\title{
GTX 2/3 EPIMERS PERMEATE THE INTESTINE THROUGH A PARACELLULAR PATHWAY
}

\author{
Rafael TORRES ${ }^{1}$, Luciano PIZARRO ${ }^{1}$, Attila CSENDES ${ }^{2}$, \\ Carlos GARCÍA ${ }^{1}$ and Néstor LAGOS ${ }^{1}$ \\ ${ }^{1}$ Lab. Bioquímica de Membrana, Dept. de Fisiología y Biofísica, \\ Facultad de Medicina, Universidad de Chile, \\ Casilla 70005, Correo 7, Santiago, Chile \\ ${ }^{2}$ Departamento de Cirugía, Hospital Clínico Universidad de Chile
}

(Received March 27, 2007; Accepted April 14, 2007)

\begin{abstract}
The aim of this work was to typify the mechanisms involved in gonyautoxins intestinal permeability. For this purpose, permeability of gonyautoxins through intestinal epithelium, and their effect on transepithelial resistance was investigated in excised human jejunal segments. The isolated mucosa segments were mounted in a Ussing chamber and experiments performed under voltage-controlled conditions. Organic gonyautoxin cations were applied in the apical side and samples collected in the basolateral side. Results show that gonyautoxin 2/3 epimers (GTX 2/3) permeate the intestine through a paracellular pathway and, to reach the resolution of the technique we used, no evidence was found of any other transport mechanism involved in the process. A model was developed, according to which tight junctions undergo a toxin concentration and time-dependent change, while transepithelial resistance shows a modest decrease.
\end{abstract}

KEY WORDS: Ussing chamber, Gonyautoxins, Permeation model, Transepithelial resistance

\section{INTRODUCTION}

Harmful algae blooms are responsible for the production of toxins that pose a threat to human lives (Lagos, 1998, 2003) and a severe detriment to the exploitation of marine resources (Hallegraeff, 1993; White, 1998). To date, six micro-algae and fresh-water cyanobacteries of associated venoms producing dissimilar clinical symptoms have been described (Yasumoto and Murata, 1993; Azevedo, 1996; Falconer, 1996; Lagos, 1998, 2003). Most of the detected toxicity is associated with Gonyaulatoxins, of which GTX $2 / 3$ epimers make up to $75 \%-80 \%$ of the toxins present in the far South of Chile (Andrinolo et al., 1999).

Gonyaulatoxins have a neurotoxic effect by selectively and reversibly blocking the voltage-sensitive Sodium channel, inhibiting action potentials. (Kao et al., 1967; Strichartz 1984; Lagos and
Andrinolo, 2000). Gonyaulatoxins exhibit different degrees of toxicity associated with their different affinities for Sodium channels (Strichartz, 1984; Oshima, 1995).

Previous work from this laboratory has shown that the toxin absorption process takes place in the small intestine at jejunal level (Andrinolo et al., 2002). It is generally accepted that the main barrier for any ingested species to enter the organism is the intestinal epithelium, and several routes can be followed to cross it. The significance of a particular transepithelial route for a given species depends on its physicochemical properties such as lipophilicity, size, hydrogen bond potential, net electrical charge and affinity for transport proteins. GTX's are chemically and structurally very similar to ribonucleosides and puric bases (Wang and Giacomini, 1997). Furthermore, at pH 7.4 they exhibit positive charge which could make them recognized by organic ion transporters of the OCT1 family found in liver and kidneys and also described in enterocytes of

Correspondence: Néstor LAGOS, Ph.D. (E-mail: nlagos@med.uchile.cl) 
the small intestine (Negel et al., 1997). Passive transport often occurs through the cell membrane of the enterocytes (transcellular transport); this is the predominant permeability route for hydrophobic compounds. Another side to the side-passive route is provided by intercellular space between enterocytes via tight junctions known as paracellular permeability. Finally, carrier-mediated transport (influx or efflux) can also be present.

Because biological membranes prevent transmembrane diffusion for most organic molecules bearing net charge, membrane-bound transport systems are generally involved in their absorption. Therefore, intestinal transporters may play a critical role in limiting and/or promoting the absorption or secretion of charged Paralytic Shellfish Poison (PSP) molecules.

The aim of this work was to typify gonyautoxin permeability at the jejunal intestinal level. Experiments were performed in a Ussing chamber under voltagecontrolled conditions. Results show that gonyautoxins cross the jejunal intestinal epithelium through a paracellular pathway.

\section{MATERIALS AND METHODS}

Toxins used in these experiments were extracted from Paralytic Shellfish Poison contaminated Mytilus chilensis collected at the southern Chilean fjords. In the filter bivalve the main paralytic shellfish toxins were the Gonyautoxin 2 and Gonyautoxin 3 epimers (GTX 2/3 epimers) (Lagos, 1996; Compagnon et al., 1998). Transepintelial potential was controlled by a World Precision Instruments (International Trade Center, 175 Sarasota Center Boulevard, Sarasota, FL 34240-9258 USA) Voltage-clamp system, model EVC4000, and its output fed into a Protek 6102A oscilloscope in order to monitor for noise or any anomalies that may go undetected by digital display of the voltage-clamp system. The signal to noise ratio was $16 \mathrm{~dB}$.

Transport experiments were carried out in a short-circuit condition in order to avoid a voltage gradient across the epithelium, because GTX 2/3 epimers have $\mathrm{a}+1$ net charge at a $\mathrm{pH}$ of 7.4 , leaving the toxin concentration gradient as the only driving force moving the toxin through the tissue. The conductance of the epithelium was obtained at all times from the $\left(\mathrm{V}_{\text {Transepithelial Potential(TEP) }}, 0\right)$ and $\left(0, \mathrm{I}_{\text {Short Circuit Cur- }}\right.$ $\operatorname{rent(SC)}$ pair of points in the V,I plane, except when the $\mathrm{I} / \mathrm{V}$ curve moved towards the vicinity of $(0,0)$ where small denominator values sent the $\mathrm{I} / \mathrm{V}$ ratio into wild swings. In those cases two points were obtained: [5, $\mathrm{I}(5)]$ and $[-5, \mathrm{I}(-5)]$, which were the first value of each pair corresponding to a transepithelial voltage imposed with the V-clamp system (in $\mathrm{mV}$ units) and the second is the current (in $\mu \mathrm{A}$ ) elicited by that potential. From these two points a straight line was obtained and its slope corresponded to the transepithelial conductance. Resistance was always calculated by taking the inverse value of conductance.

Human intestinal samples (jejune) taken from patients suffering from morbid obesity or gastric cancer subjected to total gastrectomy were used. This protocol was approved by the Ethics Committee of the University of Chile Clinical Hospital and the samples obtained with the patients signed informed consent.

Care was taken in order to avoid leaving the samples in ischemic condition. As soon as the blood vessels were clamped, the sample was cut out and submerged in a Ringer - Mannitol solution at $0^{\circ} \mathrm{C}$ gassed with a $95 \% \mathrm{O}_{2}$ and $5 \% \mathrm{CO}_{2}$ mixture and transported to the laboratory, less than a block away, where the epithelium was carefully dissected and mounted in a Ussing chamber.

\section{Solutions were as follows:}

1. Ringer Glucose: $\mathrm{NaCl} 137 \mathrm{mM}$ KCl $4.7 \mathrm{mM}$; KH2PO4 1.2 mM; NaHCO3 25 mM; MgSO4 $1.2 \mathrm{mM}$; $\mathrm{CaCl} 22.5 \mathrm{mM}$ and Glucosa $10 \mathrm{mM}$

2. Ringer Mannitol: $\mathrm{NaCl} 137 \mathrm{mM}$; $\mathrm{KCl} 4.7 \mathrm{mM}$; KH2PO4 1.2 mM; NaHCO3 25 mM; MgSO4 $1.2 \mathrm{mM}$; $\mathrm{CaCl} 22.5 \mathrm{mM}$ and Mannitol $10 \mathrm{mM}$.

Epithelia were mounted in a modified small volume Ussing chamber ( $6.8 \mathrm{ml}$ per hemichamber) with a built-in water jacket in order to circulate water at the desired temperature and a droplet trap to avoid crosscontamination by airborne droplets produced by the rupture of the gas bubbles --coming out of the stirring/ aeration system-- popping up the surface on each hemichamber.

Before mounting the tissue, at the beginning of each experimental session, reversible $\mathrm{Ag} / \mathrm{AgCl}$ electrodes purchased from World Precision Instruments, manufacturers of the voltage-clamp system, were inserted into their respective cartridge, filled with $3 \%$ Agar in a $150 \mathrm{mM} \mathrm{KCl}$ and $150 \mathrm{mM} \mathrm{NaCl}$ solution, and placed in their respective sockets in the Ussing chamber. After reaching a stable potential with RingerMannitol solution (below $2 \mathrm{mV}$ ) electrode asymmetry compensation was introduced in the voltage-clamp system. Solution conductance compensation was also introduced in order to avoid false potential readings. 
GTX 2/3 epimers paracellular pathway.

After removing the subserosa, serosa and muscle layers, the tissue was mounted as a partition between both hemichambers, the chamber was carefully mounted on its stand, solutions added at both sides and experimental procedures followed.

Control experiments demonstrated that tissue was able to maintain, and in some cases to increase, its $\mathrm{V}_{\mathrm{TEP}}$ and $\mathrm{I}_{\mathrm{SC}}$ throughout the time period required by these experiments. On each experiment, initial tissue viability was assessed by their transepithelial potential. Those with $\mathrm{V}_{\text {TEP }}<4.5 \mathrm{mV}$ were rejected.

After mounting the tissue, the apical side was bathed with Ringer-Glucose and the basolateral side was bathed with Ringer-Mannitol, both solutions at $\mathrm{pH}$ 7.4. Solutions were kept at a constant $37^{\circ} \mathrm{C}$ temperature and stirred/aerated with a flux of the gas mixture already mentioned. After $20 \mathrm{~min}$, viability was assessed, and if $\mathrm{V}_{\mathrm{TEP}}$ and $\mathrm{I}_{\mathrm{SC}}$ had reached stable values, aliquots, the volume of which depended on the final desired toxin concentration from a stock toxin concentrated solution, were added to the apical side and the experiment began.

After adding the toxin, $500 \mu \mathrm{l}$ samples were collected without replacement from the basolateral side. The first sample was taken immediately after adding the sample (time zero) and the rest of them at 20-min periods, totaling seven samples during a 120-min period. At the time of each sampling, an equal volume was sampled from the apical side in order to avoid building up a hydrostatic gradient across the epithelium.

Samples were kept frozen at $-20^{\circ} \mathrm{C}$ until their analysis by HPLC with post-column derivatization and fluorescence on line detection (Lagos, 1998).

Samples were thawed, filtered through 5000 Dalton cut Millipore Ultrafree size exclusion cartridges and concentrated by low pressure evaporation, in a Speed-Vac concentrator. After discarding the supernatant, the pellet was re-suspended in $50 \mu \mathrm{l}$ of a $25 \%$ acetic acid solution. Finally, small volume (10-20 $\mu \mathrm{l})$ samples were injected into the HPLC to be quantified.

In order to minimize random error during measurements, two different volumes of the sample were measured, providing two pairs of values $\left(\mathrm{v}_{1}, \mathrm{a}_{1}\right)$ and $\left(\mathrm{v}_{2}, \mathrm{a}_{2}\right)$, where $\mathrm{v}_{\mathrm{i}}$ and $\mathrm{a}_{\mathrm{i}}$ are the injected volumes and the related areas under the peaks corresponding to each of the epimers in the chromatogram. These two pairs of values and the default zero area at zero volume were plotted and a straight line adjusted to the plot. The same procedure was followed with the analytical toxin standards. Having the equations for each straight line, the slopes are explicitly known; therefore it is possible to determine the concentration of each epimer in the sample by using the formula

$$
\left[T_{M}\right]=\frac{m_{m}}{m_{S T}} \frac{V_{f}}{V_{i}}\left[T_{s t}\right]
$$

for a given toxin:

$\rightarrow\left[T_{M}\right]$ is the concentration of the toxin in the sample taken from the basolateral hemichamber

$\rightarrow m_{m}$ is the slope of the straight line adjusted to the values measured in the experimental sample of the toxin

$\rightarrow m_{S T D}$ is the slope of the straight line adjusted to the values measured in the analytic standard sample of the toxin

$\rightarrow \frac{V_{f}}{V_{i}}$ is the quotient between the volume of $\frac{V_{i}}{V_{i}}$ the experimental sample and its volume after concentration; in other words a concentration factor of the sample

$\rightarrow\left[T_{S T D}\right]$ is the concentration of toxin in the analytical toxin standard solution

It is important to point out that the $\mathrm{R}^{2}$ coefficient for the adjusted straight lines must be $\mathrm{R}^{2} \geq 0.98$

After quantitation of toxins at different sample collection times, the total mass amount of the epimers is quantified.

The algorithm used to calculate the amount of each epimer that has crossed the epithelium from the beginning of the experiment to the time of each sampling is:

$$
\left.M_{K}=[T x]_{K}\left[V_{0}-(k-1)\right) V_{m}\right]+V_{m} \sum_{i=1}^{K}[T x]_{i-1},
$$

with $K \in[1,6] ; K$ integer and where:

$M_{K}$ : Total mass that has crossed the epithelium from the beginning of the experiment to the time of $\mathrm{K}^{\text {th }}$ sampling

[Tx]: Toxin concentration at the moment of $\mathrm{K}^{\underline{t h}}$ sampling

$V_{0}$ : Initial volume in the hemichamber

$V_{m}$ : Volume of the sample

Algorithms to determine the $[T x]_{K}$ and $M_{K}$ were implemented in Excel $^{\circledR}$ datasheets. The same program was used to adjust a straight line to the HPLC readings in order to get the corresponding slopes for each data set.

Results for $M_{K}$ were plotted and data were adjusted to a second-order polinomial curve. The values of the R coefficients (very close to 1) support this curve of adjustment choice.

Finally, it is legitimate to consider the toxin gradient across the epithelium as a constant, because the 
amount of toxin in the basolateral side is always at least two orders of magnitude smaller than in the apical side. Therefore, using Fick's First Law does not introduce significant errors in the analysis.

\section{RESULTS}

Fig. 1 shows the time course of GTX 2 mass accumulation in the basolateral hemichamber, with data corrected for mass extraction on each sample, for different toxin concentration gradients. Fig. 2 shows the same information for GTX 3.

It is evident that both epimers accumulate at an increasing rate, and the explanation for this acceleration is that either the concentration gradient or the permeability coefficient is increasing with time. But the concentration gradient is considered constant. In fact it slowly --although not significantly-- disipates, but this dissipation would slow down the toxin flux across the epithelium and data show its acceleration. Therefore a plausible explanation is that the permeability coefficient is no longer constant, but increases with time. It is significant, however, that time flux kinetics are practically the same for both epimers, suggesting similar mechanisms and permeation pathways.

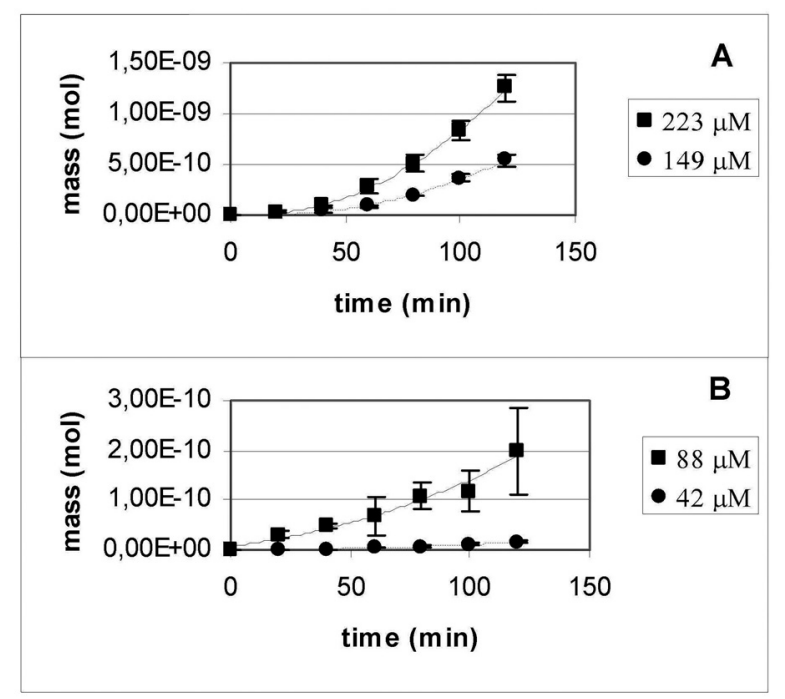

Fig. 1. GTX 2 mass accumulation in basolateral hemichamber. Plots A and B show the time course of GTX 2 mass accumulation in the basolateral hemichamber at four different concentration gradients. $\mathrm{N}=4$ for each plot. Error bars show $\pm 1 \mathrm{SD}$.
A second-order polinomial curve was adjusted on each plot, and the equation for each curve as well as its $\mathrm{R}$ coefficient are presented in Table 1.

Empirical permeability coefficient functions can be obtained from the previous equations, since the time derivative of the equations divided by the product of the experimental area of the epithelium and the concentration gradient correspond to the apparent permeability coefficient functions. Results are shown in Table 2.

Since permeabilities appear as linear functions of time and their slopes appear to change with toxin concentration, it is possible to compare permeabilities' time rate of change with time against the concentration gradient. Table 3 shows both parameters.

It is evident from the data shown in Table 3 that the permeability time rate of change increases with concentration for both epimers. Fig. 3 shows a plot based on data shown in Table 3 .

Linear functions have been fitted to data shown as straight lines in the plot. The corresponding functions and their R coefficients are shown in Table 4.

From the data exhibited, it can be concluded that:

1. Toxin flux across jejune epithelium is not a constant, but it increases with time.

2. Since the amount of toxin transferred between

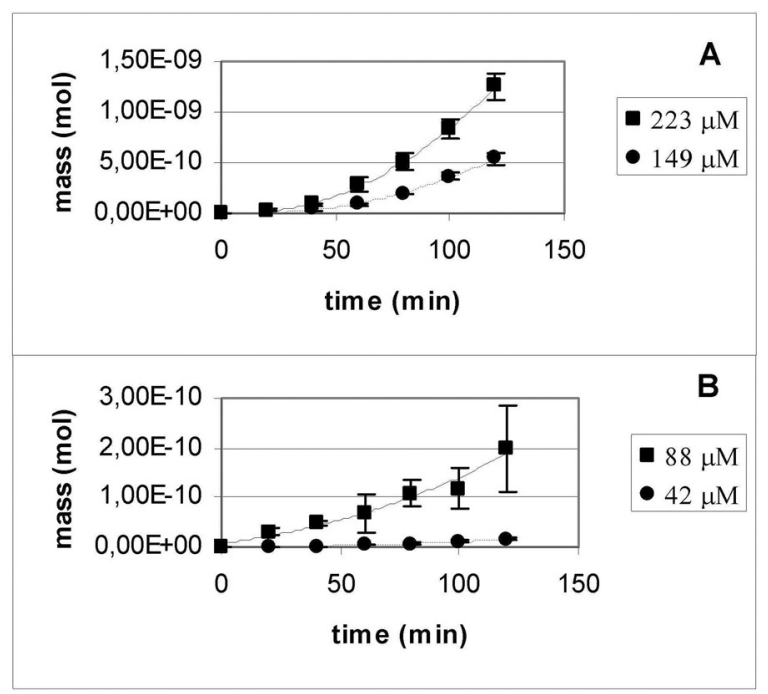

Fig. 2. GTX 3 mass accumulation in basolateral hemichamber. Plots A and B show the time course of GTX 3 mass accumulation in the basolateral hemichamber at four different concentration gradients. $\mathrm{N}=4$ for each plot. Error bars show $\pm 1 \mathrm{SD}$. 
GTX 2/3 epimers paracellular pathway.

hemichambers is very small compared with the amount in the apical side, the toxin gradient across the epithelium can be considered constant during the time experiments are carried out.

3. The only model consistent with a constant toxin gradient across the epithelium and a superlinear flux with time is one considering increasing toxin permeability with time.

4. Data show that the rate at which the permeability coefficient varies with time is a linear function of the concentration. Therefore, it can be stated that:: $P=f(t, C)$

5. The GTX 3 permeability coefficient function value is bigger than its corresponding value for GTX 2 for any $(\mathrm{t}, \Delta \mathrm{C})$ pair of values

6. An increasing permeability coefficient for these toxin molecules suggests a deterioration of the structural integrity of the epithelium. This in turn would show as a drop in the transepithelial resistance.

Conclusions (1) to (5) are summarized in Fig. 4 and 5 showing $3 \mathrm{D}$ plots of toxin permeability coefficients' dependence of time and concentration.

It seems logical to assume that while permeability increases, Transepithelial Resistance (TER) should decrease because of the apparently increasing ease for ions to cross the epithelium.

Fig. 6 shows a plot of TER versus time. It is evident that TER slightly decreased during the time period the experiments were carried out. This looks like a paradoxical result since, while TER remains almost constant, toxin permeability increases in a linear fashion with time.

\section{DISCUSSION}

Results offer clear evidence pointing to a variation of the permeability coefficients of the GTX 2/3 epimers and a first-order dependence of time and concentration gradient across the epithelium. These observed results must be paralleling structural changes, and a plausible hypothesis is to assume that toxins are changing the structural integrity of the tis-

Table 1. Toxin mass v/s Concentration gradient. Curve fitting equations for M(t). Second column shows the corresponding toxin concentration gradient and fourth column shows R, the correlation coefficient. ${ }^{\text {a Toxin con- }}$ centration gradient

${ }^{2}$ Total toxin mass transferred to basal side since toxin is added to apical side (time zero) to time " $\mathrm{t}$ ".

\begin{tabular}{|c|c|c|c|}
\hline & $\Delta \mathrm{C}^{1}(\mu \mathrm{M})$ & $\mathrm{M}(\mathrm{t})^{2}$ fitting equation $(\mathrm{mol})$ & $\mathrm{R}$ \\
\hline \multirow{4}{*}{ GTX 2 } & 223 & $\mathrm{M}(\mathrm{t})=1.000 \times 10^{-13} \mathrm{t}^{2}-1.684 \times 10^{-12} \mathrm{t}+8.944 \times 10^{-12}$ & 1.000 \\
\cline { 2 - 4 } & 149 & $\mathrm{M}(\mathrm{t})=4.942 \times 10^{-14} \mathrm{t}^{2}-1.545 \times 10^{-12} \mathrm{t}+1.550 \times 10^{-11}$ & 0.997 \\
\cline { 2 - 4 } & 88 & $\mathrm{M}(\mathrm{t})=7.806 \times 10^{-15} \mathrm{t}^{2}+5.496 \times 10^{-13} \mathrm{t}+7.938 \times 10^{-12}$ & 0.983 \\
\hline \multirow{4}{*}{ GTX 3 } & 42 & $\mathrm{M}(\mathrm{t})=1.039 \times 10^{-15} \mathrm{t}^{2}+7.118 \times 10^{-15} \mathrm{t}-1.082 \times 10^{-13}$ & 0.999 \\
\cline { 2 - 4 } & 81 & $\mathrm{M}(\mathrm{t})=3.306 \times 10^{-14} \mathrm{t}^{2}-2.810 \times 10^{-13} \mathrm{t}+3.586 \times 10^{-13}$ & 0.994 \\
\cline { 2 - 4 } & 61 & $\mathrm{M}(\mathrm{t})=1.580 \times 10^{-14} \mathrm{t}^{2}-1.017 \times 10^{-13} \mathrm{t}+2.469 \times 10^{-12}$ & 0.998 \\
\cline { 2 - 4 } & 32 & $\mathrm{M}(\mathrm{t})=4.853 \times 10^{-15} \mathrm{t}^{2}+1.457 \times 10^{-13} \mathrm{t}+8.091 \times 10^{-13}$ & 0.996 \\
\hline
\end{tabular}

Table 2. Empirical toxin permeability functions.

\begin{tabular}{|c|c|c|}
\hline & $\Delta \mathrm{C}(\mu \mathrm{M})$ & Permeability function $\left(\mathrm{cm} \mathrm{seg}^{-1}\right)$ \\
\hline \multirow{4}{*}{ GTX 2 } & 223 & $\mathrm{P}(\mathrm{t})=1.495 \times 10^{-8} \mathrm{t}-1.259 \times 10^{-7}$ \\
\cline { 2 - 3 } & 149 & $\mathrm{P}(\mathrm{t})=1.106 \times 10^{-8} \mathrm{t}-1.728 \times 10^{-7}$ \\
\cline { 2 - 3 } & 88 & $\mathrm{P}(\mathrm{t})=3.135 \times 10^{-9} \mathrm{t}-1.104 \times 10^{-7}$ \\
\cline { 2 - 3 } & 42 & $\mathrm{P}(\mathrm{t})=8.246 \times 10^{-10} \mathrm{t}-2.825 \times 10^{-9}$ \\
\hline \multirow{4}{*}{ GTX 3 } & 81 & $\mathrm{P}(\mathrm{t})=1.360 \times 10^{-8} \mathrm{t}-5.782 \times 10^{-8}$ \\
\cline { 2 - 3 } & 61 & $\mathrm{P}(\mathrm{t})=8.634 \times 10^{-9} \mathrm{t}-2.779 \times 10^{-8}$ \\
\cline { 2 - 3 } & 32 & $\mathrm{P}(\mathrm{t})=5.055 \times 10^{-9} \mathrm{t}-7.589 \times 10^{-8}$ \\
\cline { 2 - 3 } & 16 & $\mathrm{P}(\mathrm{t})=1.116 \times 10^{-9} \mathrm{t}-3.686 \times 10^{-10}$ \\
\hline
\end{tabular}

Table 3. Toxin permeability time rate of change v/s concentration.

\begin{tabular}{|c|c|c|}
\hline & $\mathrm{C}(\mu \mathrm{M})$ & $\mathrm{P}\left(\mathrm{cm} \times \mathrm{seg}^{-2}\right)$ \\
\hline \multirow{4}{*}{ GTX 2} & 223 & $1.495 \times 10^{-8}$ \\
\hline & 149 & $1.106 \times 10^{-8}$ \\
\hline & 83 & $3.135 \times 10^{-9}$ \\
\hline & 42 & $8.246 \times 10^{-10}$ \\
\hline \multirow{4}{*}{ GTX 3} & 81 & $1.360 \times 10^{-8}$ \\
\hline & 61 & $8.634 \times 10^{-9}$ \\
\hline & 32 & $5.055 \times 10^{-9}$ \\
\hline & 16 & $1.116 \times 10^{-9}$ \\
\hline
\end{tabular}


sue. An expected decrease of TER, however, does not occur, thus posing an apparent paradox, and any proposed model must account for it.

In order to develop such a model, let us assume that:

1. Tight junctions act as size exclusion filters for toxins, but they do not significantly restrict free diffusion of small ions such as Sodium,

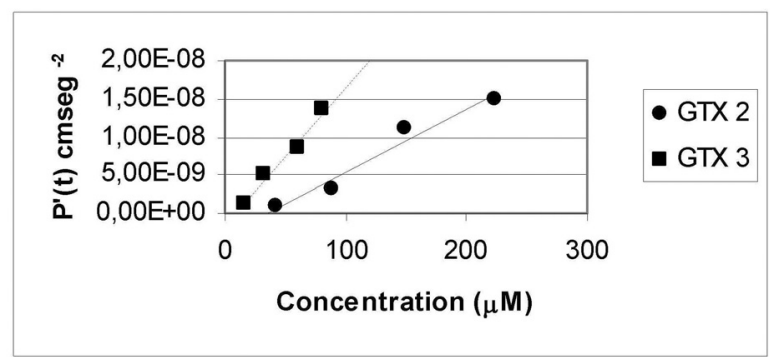

Fig. 3. Permeability time rate of change. GTX $2 / 3$ epimers' permeability time rate of change as a function of toxin concentration.

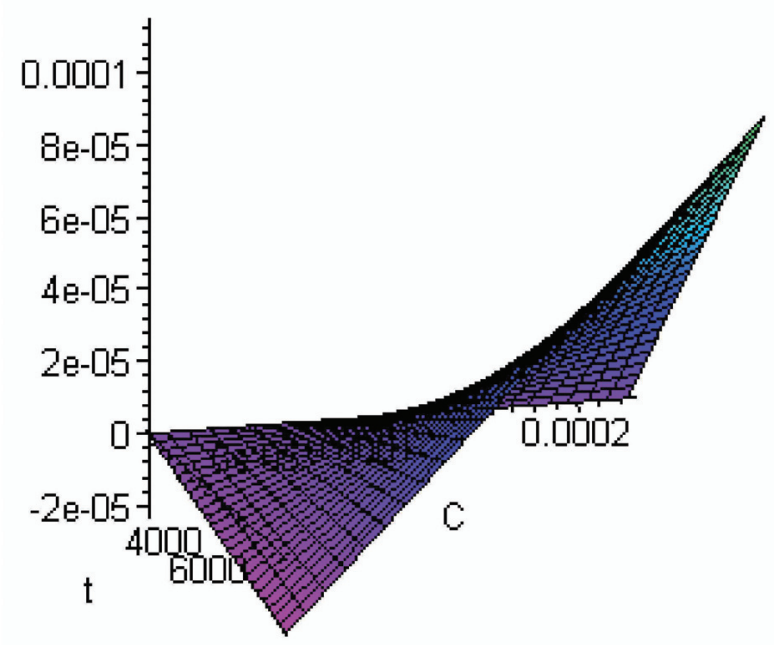

Fig. 4. GTX 2 permeability function. 3D plot showing GTX 2 permeability as a function time and toxin concentration.
Potassium and Chloride. This is a very plausible hypothesis given the difference of several orders of magnitude between atomic size ions and toxin molecules.

2. The electrolytic Ringer solution inundates every intercellular compartment, and the overall TER behaves like the equivalent resistance of a grid of many parallel resistors with

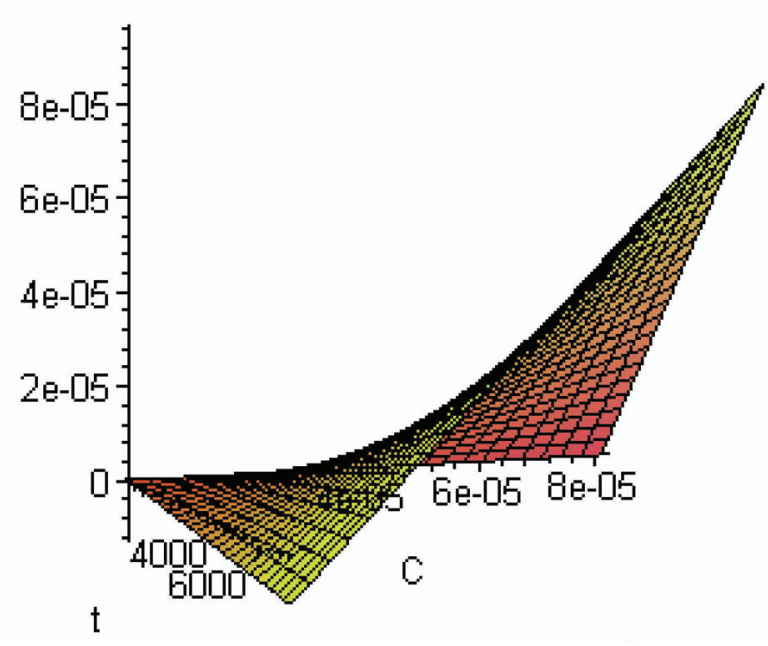

Fig. 5. GTX 3 permeability function. 3D plot showing GTX 3 permeability as a function time and toxin concentration.

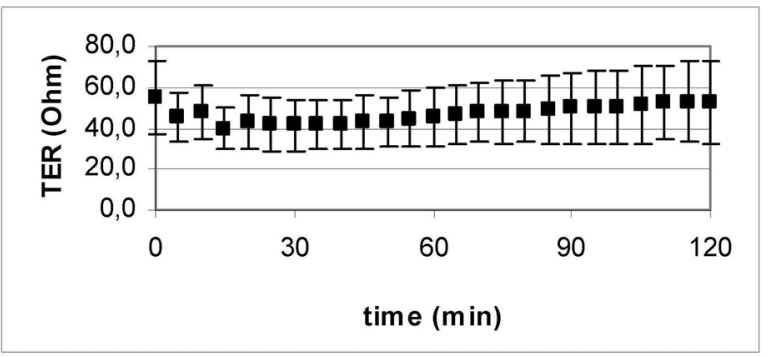

Fig. 6. Time course of transepithelial resistance. TER mean value time course during experiments. Error bars show \pm 1 SD. $N=4$.

Table 4. Linear fitting function equations.

\begin{tabular}{|c|c|c|}
\hline Toxin & Function $(\mathrm{cm} / \mathrm{seg})$ & $\mathrm{R}$ \\
\hline GTX 2 & $\mathrm{P}(\Delta \mathrm{C})=8.247 \times 10^{-11}(\Delta \mathrm{C})-2.754 \times 10^{-9}$ & 0.986 \\
\hline GTX 3 & $\mathrm{P}(\Delta \mathrm{C})=1.808 \times 10^{-10}(\Delta \mathrm{C})-1.486 \times 10^{-9}$ & 0.990 \\
\hline
\end{tabular}


GTX 2/3 epimers paracellular pathway.

a common mean value.

3. The thickness of the epithelium can be represented by a mean value in the experimental sheet placed in the Ussing chamber.

With these assumptions, the model developed (for details, see Supplemental Data) shows that the equivalent epithelium's resistance is given by

$$
R_{\text {Total }}=\frac{\rho L}{2 a D_{0} S_{0} \eta}\left[\left(1-\frac{1}{125}\right)+\left(\frac{1}{125}\right) 42\right]
$$

where:

$\rho$ : Resistivity of the Ringer solution

L: length of the paracellular pathway (approximately the thickness of the epithelium)

a: side of the base of the cells

$\mathrm{D}_{0}$ : width of the paracellular pathway

$\mathrm{S}_{0}$ : area of the experimental sheet of epithelium mounted in the Ussing chamber

$\eta$ : cell density per unit area

Ratio $1 / 125$ is the estimated ratio between the length of the paracellular pathway and the length of the tight junction (Claude and Goodenough, 1973), whereas 42 is the estimated ratio between the paracellular pathway width and the width of the tight junction at time zero (Tang and Goodenough, 2003). If toxins deteriorate the tight junctions, increasing their width up to six times their initial value, the total equivalent resistance of the epithelium will only increase a $21 \%$.

If the tight junctions act as size exclusion filters for toxin molecules, at small width values only a few molecules entering the tight junction lumen at the right position will pass through, but higher values will increase the degrees of freedom and toxin molecules will no longer be filtered out avalanching trough tight junctions, as data shows, while TER will exhibit just a small decrease.

On the other hand, the concentration gradient dependence of the permeability coefficient is coherent with the idea of a toxin attack to the tight junctions, because a simple linear diffusion would show a zero value for the permeability coefficient rate of change (i.e. a constant permeability coefficient), which clearly is not the case.

All these ideas remarkably match clinical findings. It has been observed that the higher the toxin concentration, the faster symptoms develop and the less time is available to treat patients (Lagos 2003; García et al., 2005). On the other hand, once the process has started it progressively worsens, which points towards a sustained and increasing diffusion of toxin across the epithelium.
The model accounts for the observed data. It shows that what appeared to be a paradox is properly explained when the toxin is assumed to attack tight junctions. Albeit it does not make any assumptions regarding the mechanism or nature of this attack, it shows that a sustained increase of the toxin permeability coefficient does not preclude only a modest decrease of the transepithelial electrical resistance.

It also shows that GTX 3 epimers have a permeability coefficient bigger than GTX 2, at any time and at any concentration gradient.

This model may orient future clinical procedures as well as new lines of research. Its consequences are:

1. The experimental protocol followed in this work does not allow determining whether or not there is any kind of facilitated transport of toxin across the cells, although it suggests that paracellular permeation seems to be the main transepithelial crossing way.

2. Changes in structural integrity of the intestine are a linear function of time and toxin concentration.

3. The model suggests that changes sustained by Jejune epithelium are mainly related to a direct or indirect action of toxins on the tight junctions, resulting in an increase of their lumen.

4. Linear time-dependence of toxin permeability coefficient and linear concentration dependence of the permeability time derivative suggest first-order kinetics for both variables as shown in Fig. 4.

5. The model establishes that epithelial resistance is a linear function of solution Resistivity. Therefore, although solution conductance compensation may be introduced in the Vclamp system, solution concentration changes will affect TER

6. Care must be taken when changing temperature, because solution conductivity and diffusion coefficient are both a function of temperature, and although metabolic processes leading to facilitated transport may be slowed down or even halted at low temperatures, it would be quite difficult or even impossible to discriminate between effects of temperature on conductivity, diffusion coefficients and metabolic processes.

In conclusion, this human tissue experimental preparation shows that the GTX 2/3 epimers' transepithelial transport mainly occurs by paracellular diffu- 
sion which is modeled in this study.

\section{ACKNOWLEDGMENT}

This study was supported by FEBA \# 263, Facultad de Medicina, Universidad de Chile; Organization for the Prohibition of Chemical Weapons (OPCW) Grant and FONDECYT \# 10707706. These data are part of L. Pizarro Veterinary MD Thesis.

Supplemental Data: Supplemental data are available at the on-line version of this article (http://www. jtoxsci.org).

\section{REFERENCES}

Andrinolo, D., Gomes, P., Fraga, S., Soares-da-Silva, P. and Lagos, N. (2002): Transport of the organic cations gonyautoxin $2 / 3$ epimers, a paralytic shellfish poison toxin, through the human and rat intestinal epithelia. Toxicon., 40, 13891397.

Andrinolo, D., Michea, L.F. and Lagos, N. (1999): Toxic effects, pharmacokinetics and clearance of saxitoxin, a component of paralytic shellfish poison (PSP), in cats. Toxicon., 37, 447-464.

Azevedo, S. (1996): Current studies on toxic cianobacteria (blue-green algae) of Brazilian water bodies. In IV Congreso Latino-Americano de Ficología. Caxamby, Resúmenes, p.62.

Claude, P. and Goodenough, D. A. (1973): Fracture faces of zonulae ocludentes from tight and leaky epithelium. J. Cell Biol., 58, 390-400.

Compagnon, D., Lembeye, G., Marcos, N., RuizTagle, N. and Lagos, N. (1998): Bioaccumulation of PSP toxins in bivalve Aulamomya ater and the carnivorous gastropods Concholepas and Argobuccinum ranelliformes during Alexandrium catenella bloom in the southern Chile. J. Shellfish Res., 17, 67-73.

Falconer, I.R. (1996): Potential impact on human health of toxic cianobacteria. Phycologia, 36, 611.

Garcia, C., Lagos, M., Truan, D., Lattes, K., Véjar, O., Chamorro, B., Iglesias, V., Andrinolo, D. and Lagos, N. (2005): Human intoxication with paralytic shellfish toxins: Clinical parameters and toxin analysis in plasma and urine. Biol. Res., 38, 2-3, pp.197-205. ISSN 0716-9760.

Hallegraeff, G.M. (1993): A review of harmful algal blooms and their apparent global increase. Phycologia., 32, 79-99.

Kao, C.Y., Suziki, C.Y., Kleinahus, T. and Siegman, M.J. (1967): Vasomotor and Respiratory depressant actions of tetrodotoxin and saxitoxin. Arch Int. Pharmacodyn., 165, 438-450.

Lagos, N. (1998): Microalgal blooms: A global issue whit negative impact in Chile. Biol. Res., 31, 375-386.

Lagos, N. (2003): Paralytic shellfish poisoning phycotoxins: Occurrence in South America. Comments Toxicol., 9, 1-19.

Lagos, N. and Andrinolo, D. (2000): Paralytic Shellfish Poisoning (PSP): Toxicology and Kinetics. In BOTANA LM (ed) Seafood and Freshwater Toxins: Mode of Action, Pharmacology and Physiology. pp. 203-215, Marcel Dekker, Inc., New York.

Lagos, N., Compagnon, D., Seguel, M. and Oshima, Y. (1996): Paralytic shellfish toxin composition: A quantitative analysis in Chilean mussels and dinoflagellate. Harmful and toxic algal blooms. Intergovernmental Oceanographic Commission of UNESCO. pp.121-124.

Negel, G., Volk, C., Friederich, T., Ulzheimer, J., Bamberg, E. and Koepsell, H. (1997): A revaluation of substrate specificity of the rat cation transport rOCT1. J. Biol. Biochem., 272, 471485.

Oshima, Y. (1995): Postcolumn derivatization liquid chromatographic method for paralytic shellfish toxins. J. AOAC Int., 78, 528-532.

Strichartz, G. (1984): Structural determinants of the affinity of saxitoxin in sodium channel. J. Gen Physol., 84, 281-305.

Tang, V. and Goodenough, D. (2003): Paracellular Ion Channel at the Tight Junction. Biophys. J., 84, 1660-1673.

White, A.W. (1998): Blooms of toxic algae worldwide: Their effects on fish farming and shellfish resources. Proceeding of the International Conference on the Impact of Toxic Algae on Mariculture, Aqua-Nor `87 Exhibition, pp.9-14, 1318 August 1987, Trondhein, Norway.

Wang, J. and Giacomini, K. (1997): Molecular determinants of substrate selectivity in $\mathrm{Na}^{+}$-dependent nucleoside transporters. J. Biol. Biochem., 272, 46, 28845-28848.

Yasumoto, T. and Murata, M. (1993): Marine toxins. Chem. Rev., 93, 1897-1909. 